\title{
Novel Resins for Efficient Desalination
}

Citation: T. Gettongsong, M. Taseidifar, R.M. Pashley, B.W. Ninham (2020) Novel Resins for Efficient Desalination. Substantia 4(2) Suppl.: 39-48. doi: 10.36253/Substantia-826

Copyright: () 2020 T. Gettongsong, M. Taseidifar, R.M. Pashley, B.W. Ninham. This is an open access, peer-reviewed article published by Firenze University Press (http://www.fupress.com/substantia) and distributed under the terms of the Creative Commons Attribution License, which permits unrestricted use, distribution, and reproduction in any medium, provided the original author and source are credited.

Data Availability Statement: All relevant data are within the paper and its Supporting Information files.

Competing Interests: The Author(s) declare(s) no conflict of interest.

\author{
Tanita Gettongsong ${ }^{1}$, Mojtaba Taseidifar ${ }^{1}$, Richard M. Pashley ${ }^{1, *}$, \\ BARRY W. NINHAM ${ }^{2}$ \\ ${ }^{1}$ School of Science, University of New South Wales, Canberra, Northcott Dr, Campbell \\ Australia \\ ${ }^{2}$ Department of Applied Mathematics, Research School of Physical Sciences, The Austral- \\ ian National University, Canberra, Australia \\ *Corresponding author: r.pashley@adfa.edu.au
}

\begin{abstract}
This paper reports the synthesis and properties of new polymer resins containing strong acid and base groups for optimising applications in desalination. Several polyampholytic gels were synthesised with a ratio of 1:1 of strong acid (sulphonate) and strong base (quaternary ammonium) groups and a zwitterionic resin with a 1:1 strong acid and base ratio. The physico-chemical properties of these highly charged resins were studied in electrolyte solutions over a range of $\mathrm{pH}$ values, in particular: effects of chemical cross-linking, water and electrolyte swelling; bulk electrical conductivities and surface charging properties in different $\mathrm{pH}$ values. The results from absorption of $\mathrm{NaCl}$ showed that the resins have considerable potential for more effective desalination than other resin-based techniques.
\end{abstract}

Keywords: Zwitterionic polymer resin, polyampholytic resins, desalination, ionexchange resin, ammonium bicarbonate.

\section{INTRODUCTION}

One of several themes of this Substantia volume on novel technologies for water processing concerns desalination. It has been shown that mixed cationic and anionic ion exchange resins can be used to great effect in a new desalination process. This is far more efficient in all aspects (in excess of 30\%) to the present best reverse osmosis (RO) and other techniques in use. The claim may seem extravagant. But it is the result of extensive evaluation by a major international company that builds RO plants worldwide. Our aim here is to seek to improve this new ion exchange based technology even further.

If we can build an ion exchange resin in which cationic and anionic exchange sites are on the same polymer, nanometers apart only, that in principle should do the job. Few such synthetic polymers are known. Hydrogels are composed of three-dimensional networks of polymers made of natural or synthetic materials that possess a high degree of flexibility. They have the ability to swell or de-swell, and to retain a significant fraction of water within their structure. In this study we have developed a new method to synthesise polyampholytic hydrogels containing strong acid and strong base 
ionic groups. The chemical and physical properties of highly charged hydrogels are of interest besides because of their potential as controllable shape materials. ${ }^{1}$ Shape can be controlled in several ways: by moderating the electrostatic interaction between the strong acid and base groups, their degree of hydration and hydrophobicity and also by the extent of chemical cross-linking within the resin. The range of control variables, including chemical composition make these materials of interest for their mechanical and electrical properties, their water absorption/swelling properties and their potential for selective solute separation. Especially because of the proximity of the cation and anion groups, the Hofmeister effect and other specific ion effects offer wide flexibility beyond electrostatics alone. The combination of chemical bonding as well as hydration/hydrophobic interactions and van der Waals forces offers a remarkably diverse range of materials with wide-ranging properties, and hence applications to specific ion separation. ${ }^{2,3}$

\section{THE BACKGROUND TO HYDROGELS}

By definition a hydrogel is a polymeric material which swells significantly when immersed in aqueous solution. These polymers can be covalently or ionically crosslinked to control this swelling. ${ }^{4}$ Hydrogels typically have water contents over $80 \%$ (by wt). Non-crosslinked polyampholytic compounds can show gel-like properties due to ionic cross-links that are formed by one molecule with other polymer chains, which induces enhanced plasticity and higher yield stress. These compounds will completely dissolve in aqueous concentrated salt solutions (e.g. $4 \mathrm{M} \mathrm{NaCl}$ ) at high temperatures (> 50 ${ }^{\circ} \mathrm{C}$ ), typically within days. ${ }^{1}$ This has been assumed to be because the electrostatic binding between the numerous oppositely charged groups, holding the matrix together, is weakened by the presence of an excess of oppositely charged ions in an immersing solution and also with increasing the temperature. Prima facie this is not so. Because even without salt the effective Debye length is so small that electrostatic forces will be screened. With $4 \mathrm{M}$ salt they are irrelevant. For many applications it is important to introduce controlled chemical cross-linking and swelling. The current study takes this issue on board for several hydrogels and, for comparison, a typical non-swelling polyzwitterionic resin.

Polyampholytes have already been used as additives in papermaking to improve strength. ${ }^{5}$ They are being considered for some biological replacement applications ${ }^{6}$ and for controlled drug release. ${ }^{7}$ Recently, polyampholytic hydrogels have also been employed for the efficient removal of heavy metal ions from contaminated wastewater. ${ }^{8}$ These applications are facilitated by the highly accessible open structure of these swollen polymers in water. ${ }^{9}$ It was shown first by Chandrasekar and Pashley ${ }^{10}$ that commercial strong acid and strong base mixed resins might be used to advantage for desalting water; the exhausted resin being regenerated by a process involving ammonium bicarbonate $(\mathrm{AB})$ rather than acid and base washing. ${ }^{10}$ See also other papers in this volume for detailed application.

Polyampholytic latices have small particle sizes that are similar to the polyampholytic resins. Typically, they contain weak acid and base carboxylic and tertiary amine groups. They have been recently synthesised and they show high ability to adsorb different divalent metal ions such as $\mathrm{Ca}(\mathrm{II}), \mathrm{Cd}(\mathrm{II}), \mathrm{Cu}(\mathrm{II}), \mathrm{Mg}(\mathrm{II}), \mathrm{Ni}(\mathrm{II}), \mathrm{Pb}(\mathrm{II})$ and $\mathrm{Zn}(\mathrm{II}) .{ }^{11}$ Another study has shown that these latices can exchange both cations and anions. ${ }^{12}$ Similar ionic exchange properties are found in protein molecules as well as in biomolecules, which have both cationic and anionic sites to adsorb multivalent ions of either sign. ${ }^{13-15}$ There are a variety of factors that affect ion adsorption properties of polyampholytic ion exchange resins; including $\mathrm{pH}$ of the electrolyte solution ${ }^{16,17}$, temperature $^{18,19}$, ionic strength of the electrolyte solutions ${ }^{20,21}$, the ratio of acid to basic groups and the affinities of specific counter ions. $22-24$

The polyampholytic latices are zwitterionic, and usually show a $\mathrm{pH}$ where they have net zero charge (pzc), or an isoelectric point (IEP). In order to assign an effective IEP of such latices that throws light on the behaviour of adsorption sites present on the particles, surface charge measurements and ionization models can be used. ${ }^{25-27}$

Hydrogel based compounds synthesised with 2-acrylamido-2-methylpropane sulfonic acid (AMPS) have attracted extensive attention due to their strong ionizable sulfonate group. AMPS dissociates completely over a wide $\mathrm{pH}$ range, so the hydrogels derived from it show $\mathrm{pH}$ independent swelling properties. ${ }^{28,}{ }^{29}$ Hydrogels containing amide and sulfonic groups, can form coordinate bonds with metal ions for water purification. ${ }^{30}$ Ayman et al..$^{31}$ prepared acrylamide (AM) and AMPS based hydrogel. They found that these hydrogels can take up several heavy metal ions, such as $\mathrm{Cu}$ (II), Cd (II) and Fe (III) from aqueous solutions. The recovery of hydrogels was also produced by immersion in acidic media. Yan et al. ${ }^{32}$ synthesised a series of homogeneous cross-linked uncharged and sulfonated hydrogel membranes using poly(ethylene glycol diacrylate) (PEGDA) copolymerized with AMPS. Different concentrations of sodium chloride solutions were used to determine the 
uptake of ion content $\left(\mathrm{Na}^{+}\right.$and $\left.\mathrm{Cl}^{-}\right)$based on charge density measurements on the membranes.

\section{A NOVEL DEVELOPMENT IN HYDROGELS}

All the studies mentioned above dealt only with the physical properties of the final hydrogels without considering a way to regenerate them. Therefore, interest has focused on finding novel sorbents with high adsorption capacities, fast adsorption/desorption rate, and easy separation and regeneration. The present work offers a novel regeneration process for depleted resins, using ammonium bicarbonate, that can regenerate the resins. Since it can also be readily decomposed into ammonia and carbon dioxide gases, this offers a reusable compound for the adsorption/desorption regeneration process. That is, without the need to use acid and base regeneration.

This property is unique to ammonium carbonate and is the basis of our desalination process. The work is motivated by a novel patented ion-exchange water desalination process with PCT application number: PCT/ AU2019/P110031. ${ }^{33}$

The main subject of the patent is a cross-linked organic polymer containing mixed beads of both positively and negatively charged ions at the nano scale. By integrating the positively and negatively charged ions on the one polymer, the ions are much closer together, at a nano scale, which substantially improves their absorption capacity.

The innovation relates to the application of a new, simple and low-cost method for continuously removing salt from the resin (i.e. resin regeneration process). The use of an environmentally inert ammonium bicarbonate $(\mathrm{AB})$ wash avoids the requirement for the depletion of expensive chemical reagents (i.e. acids and bases) or for heat required for resin regeneration. These are major advances.

These two innovations open up the possibility for other high-value applications of the technology, in addition to desalination applications, such as selective solute removal from contaminated water, for example, heavy metal ions and PFAS ions.

\section{MATERIALS \& METHODS}

3-(methacryloylamino) propyl-trimethylammonium chloride (MPTC) and 2-acrylamido-2-methylpropane sulfonic salt solution (AMPS) were used as strong acid cationic and strong base anionic monomers, respec- tively. Alpha-ketoglutaric acid was used as the initiator. Ethylene glycol dimethacrylate (EGDMA), and 25\% glutaraldehyde (GA) were used during the synthesis as the chemical crosslinking agent. To synthesise the zwitterionic polymer compounds N'N'-methylene bisacrylamide, PEG 400 and 1,3-propane sultone were used as monomers.

Several salts: $98 \%$ sodium chloride, $99 \%$ sodium sulphate, magnesium chloride (AR grade) and magnesium sulphate (AR grade) were used to study swelling and conductivity properties. All chemicals were used as purchased from Sigma-Aldrich, Australia without further purification. Electrical conductivity values of all the solutions were measured using a EUTECH CON 700 Conductivity Bench. A Zetasizer Nano instrument (Malvern Instruments Ltd.) was used to study the size distribution of the dry ground resin particles and the zeta-potentials of these particles dispersed in various electrolyte solutions. The chemical structures of the monomers used to produce the polyampholytic hydrogels are show in Figure 1.

The synthesised resins were characterised by microelemental analysis using Vario MICRO cube elemental analysers (Elementar Analysensysteme GmbH, Germany) and by Fourier-transform Infrared spectroscopy (FTIR) in $\mathrm{KBr}$ from $400-4000 \mathrm{~cm}^{-1}$, using a Jasco FT/ IR-6000 FTIR Spectrometer.

\section{Polyampholyte Hydrogel Synthesis Method}

Polyampholyte hydrogels were synthesized using a one-step copolymerization process. A mixed aqueous solution (monomers and initiator) was prepared and poured into the several reaction cells. It was found that glass cells with $0.5 \mathrm{~cm}$ tube diameter and $9.5 \mathrm{~cm}$ long were suitable for the polymerisation reaction. The fraction of chemicals in the reaction have been studied in different ratios, as shown in Table 1 . They were each irradiated with $365 \mathrm{~nm}$ UV light, 8 Watts, (John Morris Scientific Pty Ltd.) for $15 \mathrm{hr}$ at a distance of $5 \mathrm{~cm}$. After polymerization, the product was immersed in a large amount of water for 1 week to reach equilibrium and to wash away the residual, unreacted chemicals. Parameters were varied for the polymerization reaction; for example, the time for irradiation, distance between reaction cell and UV light source, as well as the ratio of chemical reactants and crosslinking agents. In the crosslinking processes, the product was treated by reflux reaction with glutaraldehyde and by UV copolymerization with added EGDMA.

For our study, several reaction cells were designed and developed for the polymerization reaction, as shown in Figure 2. Firstly, a rectangular metal sheet made from 


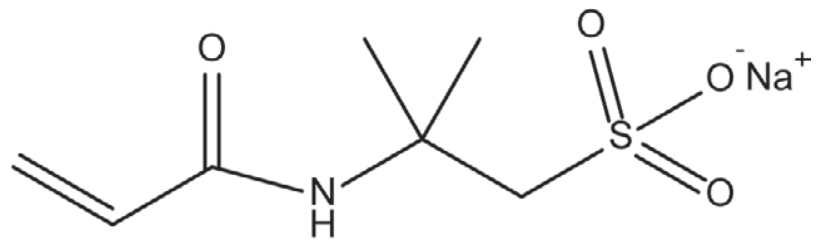

AMPS*

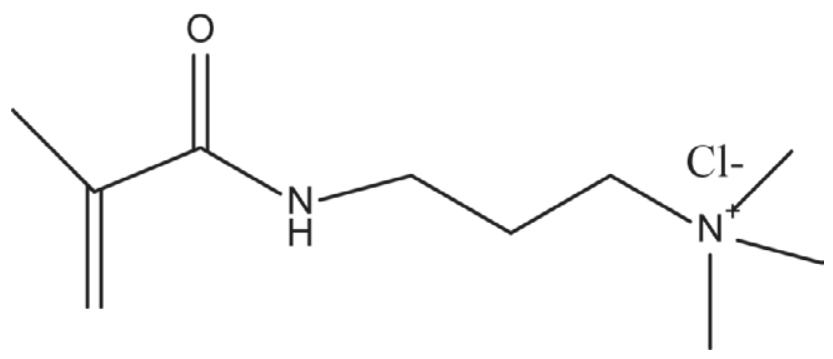

$\mathrm{MPTC}^{\star *}$

(a) Monomers for synthesis of polyampholytic hydrogel

*2-Acrylamido-2-methyl-1-propanesulfonic acid sodium salt solution

**3-(methacryloylamino) propyl-trimethylammonium chloride

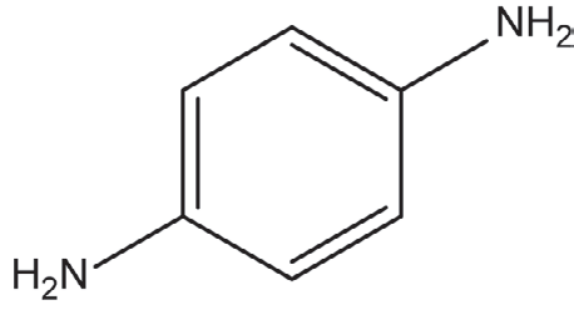

p-Phenylene diamine

(b) Reactants for synthesis of zwitterionic resins

Figure 1. Chemical structures of the monomers used to produce: (a) the polyampholytic hydrogel and (b) the zwitterionic resins.
Table 1. The ratio of monomers (molar ratio), initiator and crosslinking agent used in various synthesis reactions. In this table the initiator concentrations 1-4 refer to the ratio of monomers and $0.25 \%$ mole of initiator (i.e. for ' 1 ', and with ' 4 ' corresponding to $1 \%$ ).

\begin{tabular}{cccc}
\hline AMPS & MPTC & $\begin{array}{c}\text { 2-oxoglutaric } \\
\text { acid }\end{array}$ & EGDMA \\
\hline 1 & 1 & 1 & - \\
1 & 1 & 4 & - \\
1 & 2 & 1 & - \\
2 & 1 & 1 & - \\
1 & 1 & 1 & 1 \\
1 & 1 & 4 & 2 \\
1 & 1 & 1 & 2 \\
1 & 1 & 4 & \\
\hline
\end{tabular}

tin and an upper plate glass cover was used, as recommended in the literature. However, the results showed that tin metal also reacts with the chemicals. Therefore, an all-glass rectangular reaction cell was developed. Nevertheless, atmospheric gases still diffused into the mixture during UV polymerisation and affected the reaction. Oxygen gas is known to react with radicals and can change the polymerisation reaction. An array of glass tubes with closed-off ends to reduce gas inlet diffusion was therefore used and this was found to be the most suitable reaction cell for the UV polymerisation reaction.

\section{Zwitterionic Resin Synthesis Method}

The "zwitterionic" polymer shown in Figure 3 was synthesised using $5 \mathrm{mmol}$ of p-phenylene diamine $(0.54$ $\mathrm{g}$ ) in $20 \mathrm{~mL}$ of dimethylformamide (DMF) together with

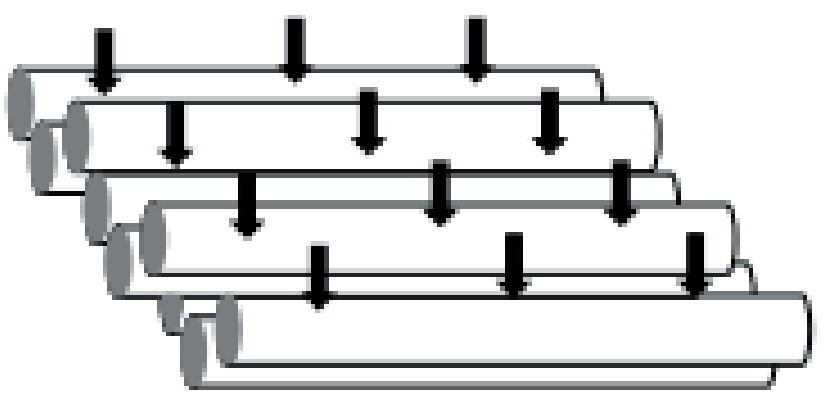

b) Array of glass tubes

a) Rectangular metal sheet

Figure 2. This schematic figure shows the different types of reaction cells that were designed and developed in the study. (a) rectangular metal and glass sheet with the reactive monomer liquid in the space between (b) an array of glass tubes with closed-off ends, with the reactive monomer liquid enclosed in the tubes. 


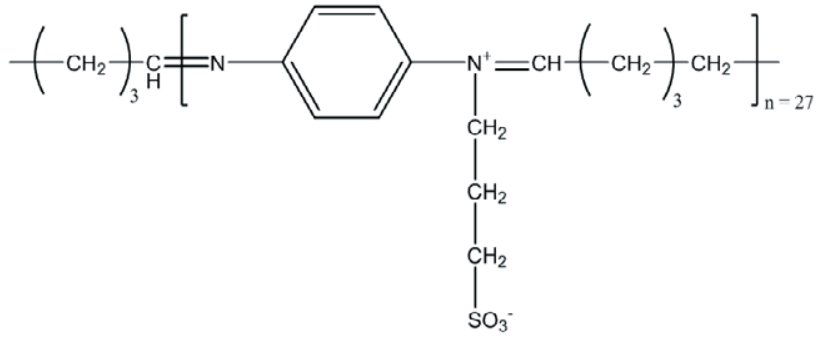

Figure 3. The chemical structure of the pseudo zwitterionic polymer studied here, as quoted $\mathrm{in}^{34}$.

$5 \mathrm{mmol}$ of glutaraldehyde $(0.47 \mathrm{~mL})$ in $20 \mathrm{~mL}$ of DMF, prepared separately in a different beaker. The solutions were mixed and refluxed at $80{ }^{\circ} \mathrm{C}$ for $1 \mathrm{hr}$. Then, $15 \mathrm{mmol}$ of 1,3 -propane sultone $(1.32 \mathrm{~mL})$ in $10 \mathrm{~mL}$ of DMF was added to the reaction and refluxed at $70{ }^{\circ} \mathrm{C}$ for $3 \mathrm{hr}$. The final product was washed several times with hot water to remove residual chemicals. The product was found to have a black gel-like form. The reason behind the colour is unclear, perhaps due to absorption of all light by the product, because of the aromatic ring of the product which has HOMO-LUMO energy gaps that absorbs light in the visible wavelengths (400-700 $\mathrm{nm})$, causing the black appearance. From the molecular diagram it is clear that the $\mathrm{C} 3$ chain connecting the sulphonate to the imine $\mathrm{N}$ cation is not of sufficient length to allow close contact between the oppositely charged groups. This supports the view that this is indeed a zwitterionic polymer. However, it should also be realised that this polymer is not actually a pure zwitterionic polymer in any case because of the presence of a second imine group, which will readily become protonated in aqueous solutions below $\mathrm{pH} 10$. This is due to the $\mathrm{pK}_{\mathrm{a}}$ value of the imine group, which is around 10. In typical aqueous solutions this polymer will therefore actually be a $2+/ 1$ - ionic polymer and on dissolution will also act to increase the solution $\mathrm{pH}$.

\section{Powdered Resin Samples Preparation}

The products of the various polyampholyte hydrogels and the zwitterionic resin compound were allowed to completely dry in a fume cupboard at room temperature. A mortar and pestle dry-grinding system was used to produce finely ground particle samples of each dry resin. The resins were all in the water-washed state, prior to drying, to maintain maximum electrostatic binding of the polymer matrix and hence solid rigidity, to enhance dry-grinding efficiency.

\section{FT-IR Results}

The FT-IR spectra was obtained for both resin samples and are given in Figure 4. Broad absorptions

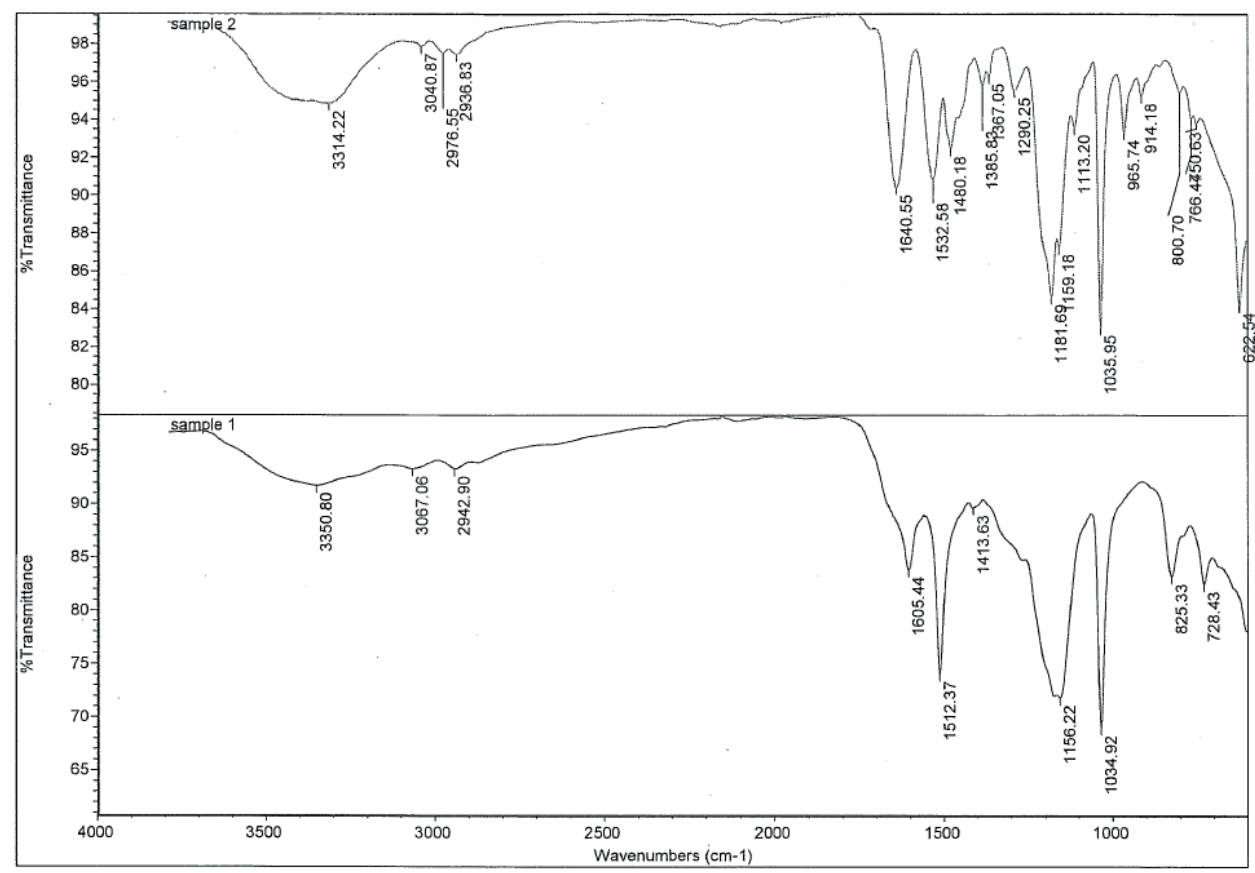

Figure 4. FT-IR spectra for zwitterionic resin (Sample 1) and hydrogel resin (Sample 2). 
around $3400 \mathrm{~cm}^{-1}$ indicate free $\mathrm{O}-\mathrm{H}$. The absorptions at $2900-3000 \mathrm{~cm}^{-1}$ are due to the $\mathrm{C}-\mathrm{H}$ asymmetric stretch. The carboxybetaine was characterised by absorptions at $1181 \mathrm{~cm}^{-1}$ (C-CO-C) stretched band in Sample 2 for the hydrogel resin. Also $1640 \mathrm{~cm}^{-1}$ indicates $\mathrm{C}=\mathrm{O}$ stretching in this sample. The aromatic ring stretching absorptions $(\mathrm{C}=\mathrm{C}$ and $\mathrm{C}=\mathrm{N})$ for the Sample 1 (zwitterionic resin) can be seen in the range $1413-1605 \mathrm{~cm}^{-1}$. The characteristic absorption for sulfonate groups present in both resins, appear at $1034 \mathrm{~cm}^{-1}, 1035 \mathrm{~cm}^{-1}$ and $1640 \mathrm{~cm}^{-1}$, which are highly intense. ${ }^{34}$

\section{RESULTS AND CHARACTERISATION}

Several polyampholytic hydrogels, produced without chemical crosslinking, were made from a ratio of 1:1 (AMPS: MPTC) with initiator. These resins readily produced a clear film on drying but were also easily dispersed in water, losing their structure, as illustrated in the example in Figure 5.

Several polyampholytic hydrogels were formed with chemical crosslinking using EGDMA and GA. These were added to the polymerisation reaction mixture to
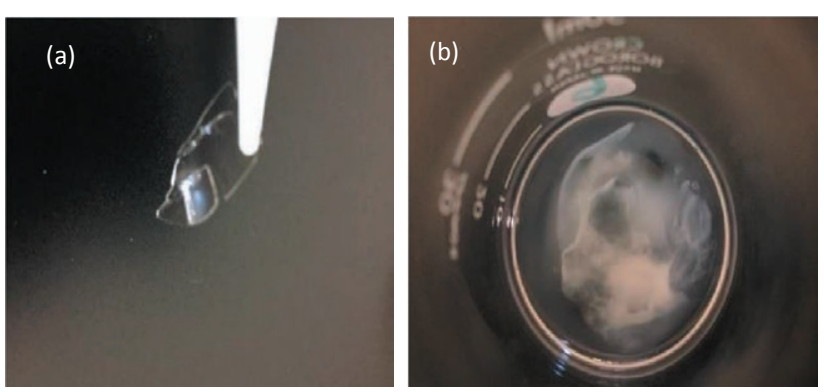

Figure 5. These photographs illustrate that the polyamplolytic hydrogels formed without the use of chemical crosslinking agents formed clear plastic films when dry (a) and then when equilibrated with excess water (b), the polymer chains completely dispersed.

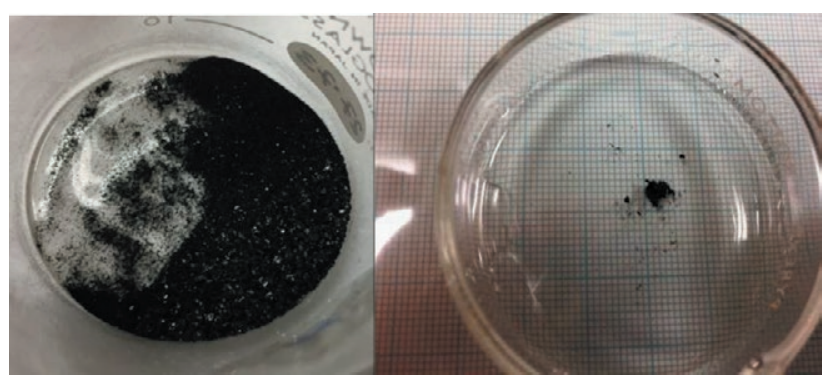

Figure 6. The product of the zwitterionic synthesis dry product (on left) and the after 24 hours water-swelling (on right). reduce the aqueous swelling properties of the hydrogels. An example of a suitable ratio of polyampholyte hydrogel with crosslinking agent is $1: 1: 2: 1$ (AMPS : MPTC : EGDMA : initiator ). Note that in this scheme ' 1 ' for the initiator refers to a level of $0.25 \%$. The polymerisation results obtained show that chemically crosslinked hydrogels prevent the release of the constituent polymer chains when immersed in water, while still allowing substantial aqueous uptake or swelling.

By comparison, the zwitterionic polymer containing similar charged groups, that is sulphonate and quaternary ammonium groups, produced a black powder which showed little or no swelling in water, as illustrated in Figure 6. The results of a swelling study showed that both zwitterionic polymer products did not show any significant swelling in either pure water or a range of electrolyte solutions.

These observations are consistent with the extent of chemical cross-linking expected in the final product and the hydrophobicity of these polymers. A qualitative evaluation of the relative hydrophobicity of the powdered resin samples can be achieved using a simple 'water float test'. Small amounts of both the cross-linked hydrogel (dry ground into powder) and the zwitterionic resin (dry ground) powder were carefully sprinkled onto the surface of water. These observations indicate that the zwitterionic resin is much more hydrophobic, which is also consistent with its lack of swelling when immersed in water. By comparison, the hydrogel resin both swells substantially in water and is water wet, and so readily enters the aqueous phase.

The finely dry-ground resin samples were dispersed by simple stirring in water and $1 \mathrm{mM} \mathrm{NaCl}$ solution at room temperature and the particle size distributions were measured using a Malvern Zetasizer light scattering instrument (model ZS). In addition, the zeta potentials of the dispersed particles of each resin were measured in $1 \mathrm{mM} \mathrm{NaCl}$ solution, at room temperature. Typical results obtained are shown in Table 2 .

The elemental analysis for two polymer resins show similar results for $\mathrm{N}, \mathrm{H}$ and $\mathrm{S}$ atoms in their chemical structures, except that the zwitterionic resin had a higher level of carbon compared to the hydrogel resin. This is because the zwitterionic sample comprises a higher level of carbon in its aromatic rings. These results are averaged based on two analyses for each sample and the average values are given in Table 3.

A summary of typical results obtained using the two powdered resin samples dispersed in $1 \mathrm{mM} \mathrm{NaCl}$ in different $\mathrm{pH}$ values at room temperature is given in Table 2 . The results indicate that the polyampholytic particles are typically clumped together in water. This cannot be due to electrostatic binding between the positive and negative charged groups on facing polymer particles 
Table 2. Particle size distribution (radius in $\mathrm{nm}$ ) and zeta potential $(\mathrm{mV})$ of the two polymer resins in different $\mathrm{pH}$ solutions. Note that all the samples were prepared in $1 \mathrm{mM} \mathrm{NaCl}$ solution and $\mathrm{pH}$ adjusted using $0.1 \mathrm{M} \mathrm{NaOH}$ and $0.1 \mathrm{M} \mathrm{HCl}$ solution.

\begin{tabular}{ccccc}
\hline \multirow{2}{*}{$\mathrm{pH}$} & \multicolumn{2}{c}{ Size $(\mathrm{r} / \mathrm{nm})$} & \multicolumn{2}{c}{ Zeta potential $(\mathrm{mV})$} \\
\cline { 2 - 5 } & Hydrogel & Zwitterionic & Hydrogel & Zwitterionic \\
\hline 3 & 915 & 3849 & -8.79 & -5.65 \\
6 & 1194 & 14700 & -9 & -19.2 \\
9 & 9142 & 9180 & -15.9 & -17.5 \\
\hline
\end{tabular}

Table 3. Total elemental analysis of two synthesised polymer resins.

\begin{tabular}{ccccc}
\hline & $\% \mathrm{C}$ & $\% \mathrm{H}$ & $\% \mathrm{~N}$ & $\% \mathrm{~S}$ \\
\hline Zwitterionic & 53.44 & 5.81 & 8.98 & 5.40 \\
Hydrogel & 43.92 & 8.60 & 8.69 & 6.24 \\
\hline
\end{tabular}

alone and must involve some specific hydration effects. It is known from extensive work on microemulsions, force measurements between surfactant bilayers and NMR that the quaternary ammonium group has two tightly bound water molecules of hydration. Bromide, chloride, iodide and fluoride all bind strongly, and the ion pair is effectively neutral. These anions displace divalent sulphate ions. On the other hand sodium binds very strongly to the sulphonate group whereas other cations do not. So we expect that addition of $\mathrm{NaCl}$ to the dispersion will weaken this inter-polymer-polymer binding, due to reduced hydration and polymer bridging forces. Polymer swelling could not cause the large particle size differences seen in Table 2.

Addition of salt should increase the swollen size of individual particles, as reported by Kudaibergenov and Ciferri, 2013. ${ }^{9}$ But as for above the effect can be expected to be ion specific. Osmotic pressure effects caused by the $1 \mathrm{mM} \mathrm{NaCl}$ solution will only be about $0.05 \mathrm{~atm}$, which is too small to cause any significant dewatering of the resin particles. The standard argument for the overall negative zeta potential of the hydrogel particles in $1 \mathrm{mM}$ $\mathrm{NaCl}$ solution, shown in Table 2, supposes that this is expected for polymers with similar densities of positive and negative charged groups because the $\mathrm{Na}^{+}$ion is more strongly hydrated than the $\mathrm{Cl}^{-}$ion and so is less readily adsorbed onto the particle surface.

Biological cells and the common, natural inorganic particles, such as quartz and clays, are negatively charged for the same reason. However the classical theory is erroneous but usually reasonable at very low salt. ${ }^{35-}$ ${ }^{37}$ We persist with the classical colloid science approach keeping in mind that it can be misleading.
The zwitterionic resin particles were found to have a lower (in magnitude) negative zeta potential, see Table 2 . This is consistent with the additional imine group present on this zwitterionic molecule (see Figure 3). It will protonate, depending on $\mathrm{pH}$, and so should reduce the overall negative potential.

A comparison of water swelling of the cross-linked (1:1:2:1 sample) polyampholytic hydrogel in pure water and in various $0.2 \mathrm{M}$ electrolyte solutions was studied visually. The solutions were equilibrated for $24 \mathrm{hr}$ at room temperature. The results showed that water content was typically found to be around $90 \%$ for chemically cross-linked gels immersed in a range of electrolyte solutions. These swelling results indicate that pure water produced the greatest swelling and that all of the salts reduced the swelling to a similar extent relative to pure water. And that there appeared to be no significant specific ion effects on the degree of swelling, even when the osmotic pressure of the solution was increased, say for $\mathrm{MgCl}_{2}$ and $\mathrm{Na}_{2} \mathrm{SO}_{4}$ solutions. At least the differences between the monovalent sodium and divalent magnesium are not significant. (That need not be so for other cations like nickel, or anions in the Hofmeister series that have not yet been tested.)

\section{CONDUCTIVITY VALUES}

Bulk electrical conductivities of the swollen gels were measured and compared with bulk solution values for: water, $\mathrm{NaCl}$ and $\mathrm{MgSO}_{4}$ solutions, over a 3-day period. These results, given in Tables 4-7, showed that the conductivity was reduced in the swollen gels by around 10 percent for each solution. In other words, there appeared to be no specific ion effects and the gels with a high-water content, gave electrical conductivities, even the two different electrolytes, roughly consistent with their water volume fraction. These results reflect the high-water content of these hydrogels.

That these single polymer resins can make useful ion exchange resins is clearly demonstrated by their ability to absorb $\mathrm{NaCl}$ from solution, as shown by the results given in Table 4. The absorption trend shows that the zwitterionic resin offered a significant absorption capacity from 1 to $28 \mathrm{mmol} / \mathrm{g}$ for $\mathrm{NaCl}$ solution from $0.1 \mathrm{M}$ to $0.3 \mathrm{M}$, respectively. While the hydrogel resin absorption capacity increased from 4 to $9 \mathrm{mmol} / \mathrm{g}$ for the two $\mathrm{NaCl}$ solutions.

These results compare favourably with the most efficient commercially available, mixed-bed, strong acid and strong base resin systems, which absorb $\mathrm{NaCl}$ from aqueous solution at a level typically of about $2.5 \mathrm{mmol} / \mathrm{g}$. 
Table 4. Electrical conductivity results for the hydrogel polymer dry sample (weight $0.05 \mathrm{~g}$ ) in $50 \mathrm{~mL} \mathrm{NaCl}$ solution (with an average experimental error of about $\pm 0.05 \mathrm{mS} / \mathrm{cm}$ ).

\begin{tabular}{ccc}
\hline \multirow{2}{*}{ Concentration $(\mathrm{M})$} & \multicolumn{2}{c}{ conductivity $(\mathrm{mS} / \mathrm{cm})$} \\
\cline { 2 - 3 } & Before & after \\
\hline 0.2 & 19.1 & 18.7 \\
0.25 & 23.2 & 22.8 \\
0.3 & 27.3 & 26.4 \\
0.4 & 34.9 & 33.7 \\
0.5 & 41.3 & 40.5 \\
\hline
\end{tabular}

Table 5. Electrical conductivity results for the zwitterionic polymer sample (weight $0.05 \mathrm{~g}$ ) in $50 \mathrm{~mL} \mathrm{NaCl}$ solution (with experimental error $\pm 0.05 \mathrm{mS} / \mathrm{cm})$.

\begin{tabular}{ccc}
\hline \multirow{2}{*}{ Concentration $(\mathrm{M})$} & \multicolumn{2}{c}{ conductivity $(\mathrm{mS} / \mathrm{cm})$} \\
\cline { 2 - 3 } & Before & after \\
\hline 0.1 & 10.4 & 10.3 \\
0.3 & 27.5 & 24.8 \\
0.5 & 35.4 & 34.1 \\
\hline
\end{tabular}

Table 6. Electrical conductivity results for $0.2 \mathrm{M}$ salt solutions (Conductivity in solution) with experimental error $\pm 0.05 \mathrm{mS} / \mathrm{cm}$.

\begin{tabular}{ccccc}
\hline & \multicolumn{4}{c}{ Conductivity $(\mathrm{mS} / \mathrm{cm})$} \\
\cline { 2 - 5 } & day 0 & day 1 & day 2 & day 3 \\
\hline $\mathrm{NaCl}$ & 18.9 & 18.7 & 18.6 & 18.4 \\
$\mathrm{MgSO}_{4}$ & 15.9 & 15.9 & 15.8 & 15.7 \\
$\mathrm{DI}$ water & 0.002 & 0.013 & 0.04 & 0.05 \\
\hline
\end{tabular}

Table 7. Electrical conductivity results for $0.2 \mathrm{M}$ salt solutions (Conductivity in gel) with experimental error $\pm 0.05 \mathrm{mS} / \mathrm{cm}$.

\begin{tabular}{ccccc}
\hline & \multicolumn{4}{c}{ Conductivity $(\mathrm{mS} / \mathrm{cm})$} \\
\cline { 2 - 5 } & day 0 & day 1 & day 2 & day 3 \\
\hline $\mathrm{NaCl}$ & & 15.6 & 16.7 & 16.3 \\
$\mathrm{MgSO}_{4}$ & 13.5 & 13.7 & 14.0 \\
$\mathrm{DI}$ water & 0.04 & 0.04 & 0.06 \\
\hline
\end{tabular}

\section{ADDITIONAL COMMENTS}

In the course of this work it was realised that the 'zwitterionic' polymeric resin compounds containing amide groups reported by Tarannum and $\operatorname{Singh}^{34}$ have been erroneously defined as zwitterionic. In fact, they are anionic resins only. This is because the nitrogen atom in
Table 8. The resin absorption capacities $(+/-0.5 \mathrm{mmol} / \mathrm{g})$ estimated from the measured absorption from aqueous $\mathrm{NaCl}$ solutions (i.e. 0.1 and $0.3 \mathrm{M})$.

\begin{tabular}{ccc}
\hline \multirow{2}{*}{ Resin } & \multicolumn{2}{c}{$\mathrm{mmol} / \mathrm{g}$ of absorption } \\
\cline { 2 - 3 } & $0.1 \mathrm{M}$ & $0.3 \mathrm{M}$ \\
\hline Hydrogel & 4.3 & 9.3 \\
Zwitterionic & 1.0 & 28.1 \\
\hline
\end{tabular}

the amide group will not be protonated under normal solution conditions. It can actually be protonated only in very strong acid solution. That is, the zwitterionic structure reported in the literature can only be formed in very strong acid solution. For all practical uses the resin acts as an anionic, sulphonated, resin. The $\mathrm{pK}_{\mathrm{a}}$ of the conjugate acid nitrogen in an amide group present in the hydrogel resin, is about -0.5 , which means that an acid with $3 \mathrm{M}$ concentration is required to protonate the amide group in this resin to form a zwitterionic compound. Adsorption isotherms for the zwitterionic resins showed a maximum $\mathrm{NaCl}$ adsorption of about $28 \mathrm{mmol} / \mathrm{g}$ (dry wt.), while for the same concentration of $\mathrm{NaCl}$, hydrogel resins had adsorption levels of about $9 \mathrm{mmol} / \mathrm{g}$ (dry wt.). In addition, as also mentioned earlier, the other zwitterionic compound, used in this work, will have a protonated imine group in most aqueous solutions, in addition to the zwitterionic (sulphate/quaternary ammonium) group. Hence, this compound is also not a 'true' zwitterionic polymer.

This work was designed to extend the efficiency of a novel patented ion-exchange water desalination process. ${ }^{33}$ In this patent, ammonium bicarbonate (AB) solution has been used to regenerate depleted mixed bed ion exchange resins for subsequent use in desalinating salt solutions. A bubble column evaporator (BCE) can then be used to decompose the $\mathrm{AB}$ product solution into drinking water, ammonia $\left(\mathrm{NH}_{3}\right)$ and carbon dioxide $\left(\mathrm{CO}_{2}\right)$ gases; this is the subject of another work published in this special issue of Substantia. The gases can then be collected into a cool aqueous solution for reuse in further regenerating the resin.

A commercial-in-confidence report on this patent was prepared by the international engineering company Arcadis for Breakthrough Water Technology on behalf of a major International Gold Mining Group. The results in this report show that this method is likely to be up to $30 \%$ more efficient and less energy consuming than current reverse osmosis (RO) and ion-exchange desalination processes. We will be working on this method to scale up the technique for commercial usage. This project aims to establish in a larger scale pilot unit for further testing, evaluation and development. 


\section{CONCLUSIONS}

A new UV method to produce polyampholytic hydrogels was developed. It was found that it is very difficult to chemically cross-link the gels to reduce swelling in water. Interestingly, the gels have a high-water content and their electrical conductivities, even in different electrolytes, are consistent with their water volume fraction. Strong acid and strong base polyampholytic gels have some unusual chemical and physical properties. We also found that some zwitterionic resins reported in the literature have been erroneously classified. The results are encouraging. There is clear advantage for desalination applications for a resin with cationic and anionic ion exchange sites angstroms apart on the same polymer. By comparison, with conventional mixed cationic and anionic beads, presently available and evaluated and proposed for desalination, as is outlined in this volume, the oppositely charged groups can be millimeters apart. While we have seen in earlier papers that the mixed cationic and anionic resins are much more efficient than reverse osmosis, the new structures would be more efficient still. Some further study to confirm the robustness of the regeneration process is necessary to confirm its expected successful availability as an efficient desalination system.

A Final Comment. Because of the very large variation in specific ion binding capacities of both the quaternary ammonium and sulphonate moieties of the gel it might be expected, that combined with the ammonium bicarbonate process, it might well have applications beyond ordinary desalination per se.

There are very major problems with natural drinking water contamination with fluoride. and the perennial problem of nitrate and phosphate ions in runoff water in agriculture.

We hope to tackle these issues subsequently.

\section{ACKNOWLEDGMENTS}

The authors would like to thank Dr. Mokhlesur Rahman for his support and suggestions on the synthesising procedures. The authors also would like to thank Dr. Remi Rouquette of Macquarie University for the elemental analysis and the FTIR analysis.

\section{REFERENCES}

1. T.L. Sun, T. Kurokawa, S. Kuroda, A.B. Ihsan, T. Akasaki, K. Sato, M.A. Haque, T. Nakajima, J.P. Gong,
Physical hydrogels composed of polyampholytes demonstrate high toughness and viscoelasticity, Nat Mater, 2013, 12(10), 932-7.

2. L. Su, S. Khan, J. Fan, Y.-N. Lin, H. Wang, T.P. Gustafson, F. Zhang, K.L. Wooley, Functional sugarbased polymers and nanostructures comprised of degradable poly(d-glucose carbonate)s, Poly. Chem., 2017, 8(10), 1699-1707.

3. D. Tatini, F. Sarri, P. Maltoni, M. Ambrosi, E. Carretti, B.W. Ninham, P. Lo Nostro, Specific ion effects in polysaccharide dispersions, Carbohydr. Polym., 2017, 173, 344-352.

4. C.A. Finch, Polymers in aqueous media: Performance through association. Advances in Chemistry Series No. 223 Edited by J. E. Glass, ACS, Washington, Polym. Int., 1991, 25(1), 61-62.

5. M.A. Hubbe, O.J. Rojas, D.S. Argyropoulos, Y. Wang, J. Song, N. Sulić, T. Sezaki, Charge and the drystrength performance of polyampholytes: Part 2 . Colloidal effects, Colloids Surf., A:Physiochem. Eng. Aspects, 2007, 301(1), 23-32.

6. A.B. N. Alepee, M. Daneshian, B. De Wever, E. Fritsche, A. Goldberg, J. Hansmann, T. Hartung, J. Haycock, H.T. Hogberg, t4 workshop report: State-ofthe-art of 3D cultures (organs-on-a-chip) in safety testing and pathophysiology, Altex, 2014, 31(4), 441-477.

7. J. H. Chen, C. C. Tsai, Y.Z. Kehr, L. Horng, K. Chang, L. Kuo, An Experimental Study of Drag Reduction in a Pipe with Superhydrophobic Coating at Moderate Reynolds Numbers, in ICEM 14 - 14th International Conference on Experimental Mechanics. EPJ Web of Conferences Poitiers, France, 2010.

8. C. Zhang, C. Lai, G. Zeng, D. Huang, C. Yang, Y. Wang, Y. Zhou, M. Cheng, Efficacy of carbonaceous nanocomposites for sorbing ionizable antibiotic sulfamethazine from aqueous solution, Water Res., 2016, 95, 103-112.

9. S.E. Kudaibergenov, A. Ciferri, Natural and Synthetic Polyampholytes, 2, Macromol. Rapid Commun., 2007, 28(20), 1969-1986.

10. N.P.G.N. Chandrasekara, R.M. Pashley, A model for ion-exchange behaviour of polyampholytic resins: Using polystyrene polyampholytic latex, Colloids Surf., A:Physiochem. Eng. Aspects, 2017, 516, 39-47.

11. D.S. Eldridge, R.J. Crawford, I.H. Harding, The role of metal ion-ligand interactions during divalent metal ion adsorption, J. Colloid Interface Sci., 2015, 454, 20-26.

12. A. Homola, R.O. James, Preparation and characterization of amphoteric polystyrene latices, J. Colloid Interface Sci., 1977, 59(1), 123-134.

13. E. Ruckenstein, M. Manciu, Stability of dispersions, in Nanodispersions: Interactions, Stability, and 
Dynamics, Springer New York: New York, NY, 2010, 201-324.

14. S. Salgin, U. Salgin, S. Bahadir, Zeta potentials and isoelectric points of biomolecules: The effects of ion types and ionic strengths, Int. J. Electrochem. Sci., 2012, 7(12), 12404-12414.

15. S. Perez-Amodio, P. Holownia, C.L. Davey, C.P. Price, Effects of the ionic environment, charge, and particle surface chemistry for enhancing a latex homogeneous immunoassay of C-reactive protein, Anal. Chem., 2001, 73(14), 3417-3425.

16. I.H. Harding, T.W. Healy, Adsorption of aqueous cadmium(II) on amphoteric latex colloids: I. General kinetics and thermodynamics, J. Colloid Interface Sci., 1985, 107(2), 362-370.

17. I.H. Harding, T.W. Healy, Adsorption of aqueous cadmium(II) on amphoteric latex colloids: II. Isoelectric point effects, J. Colloid Interface Sci., 1985, 107(2), 371-381.

18. M. Chanda, S.A. Pillay, A. Sarkar, J.M. Modak, A thermally regenerable composite sorbent of crosslinked poly(acrylic acid) and ethoxylated polyethyleneimine for water desalination by Sirotherm process, J. Appl. Polym. Sci., 2009, 111(6), 2741-2750.

19. N.P.G.N. Chandrasekara, R.M. Pashley, Study of a new process for the efficient regeneration of ion exchange resins, Desalination, 2015, 357, 131-139.

20. B.A. Bolto, R. McNeill, A.S. MacPherson, R. Siudak, D.E. Weiss, D. Willis, An Ion-Exchange Process with Thermal Regeneration. VI. Factors Influencing the Titration Curve Shape of Weak Electrolyte Resins, Australian J. Chem., 1968, 21(11), 2703-2710.

21. D.E. Weiss, B.A. Bolto, R. McNeill, A.S. MacPherson, R. Siudak, E.A. Swinton, D. Willis, An Ion-Exchange Process with Thermal Regeneration. IV. Equilibria In A Mixed Bed of Weak-Electrolyte Resins, Australian J. Chem., 1966, 19(5), 765-789.

22. D. Weiss, B. Bolto, R. McNeill, A. MacPherson, R. Siudak, E. Swinton, D. Willis, An ion-exchange process with thermal regeneration. II. Properties of weakly basic resins, Australian J. Chem., 1966, 19(4), 561-587.

23. D.E. Weiss, B.A. Bolto, R. McNeill, A.S. MacPherson, R. Siudak, E.A. Swinton, D. Willis, An IONExchange Process with Thermal Regeneration. III. Properties of Weakly Acidic ION-Exchange Resins, Australian J. Chem., 1966, 19(4), 589-608.

24. V.K. Koul, A.K. Gupta, Uptake of sodium chloride by mixture of weakly acidic and weakly basic ion exchange resins: equilibrium and kinetic studies, Chem. Eng. Sci., 2004, 59(7), 1423-1435.

25. J.T. Duniec, J.N. Israelachvili, B.W. Ninham, R.M. Pashley, S.W. Thorne, An ion-exchange model for thylakoid stacking in chloroplasts, FEBS Letters, 1981, 129(2), 193-196.

26. R.M. Pashley, DLVO and hydration forces between mica surfaces in $\mathrm{Li}^{+}, \mathrm{Na}^{+}, \mathrm{K}^{+}$, and $\mathrm{Cs}^{+}$electrolyte solutions: A correlation of double-layer and hydration forces with surface cation exchange properties, $J$. Colloid Interface Sci., 1981, 83(2), 531-546.

27. F. Makavipour, R.M. Pashley, A study of ion adsorption onto surface functionalized silica particles, Chem. Eng. J., 2015, 262, 119-124.

28. S. Durmaz, O. Okay, Acrylamide/2-acrylamido2-methylpropane sulfonic acid sodium salt-based hydrogels: synthesis and characterization, Polym., 2000, 41(10), 3693-3704.

29. L. Zhang, A. Eisenberg, Formation of crew-cut aggregates of various morphologies from amphiphilic block copolymers in solution, Polym. Adv. Technol.s, 1998, 9(10-11), 677-699.

30. A. El-Hag Ali, H.A. Shawky, H.A. Abd El Rehim, E.A. Hegazy, Synthesis and characterization of PVP/ AAc copolymer hydrogel and its applications in the removal of heavy metals from aqueous solution, Europ. Polym. J., 2003, 39(12), 2337-2344.

31. A.M. Atta, H.S. Ismail, A.M. Elsaaed, Application of anionic acrylamide-based hydrogels in the removal of heavy metals from waste water, J. Appl. Polym. Sci., 2012, 123(4), 2500-2510.

32. N. Yan, D.R. Paul, B.D. Freeman, Water and ion sorption in a series of cross-linked AMPS/PEGDA hydrogel membranes, Polym., 2018, 146, 196-208.

33. R.M. Pashley, M. Taseidifar, T. Gettongsong, Resin for desalination and process of regeneration, PCT, Google Patents, 2019.

34. N. Tarannum, M. Singh, Synthesis and characterization of zwitterionic organogels based on Schiff base chemistry, J. Appl. Polym. Sci., 2010, 118(5), 28212832.

35. B.W. Ninham, R.M. Pashley, P. Lo Nostro, Surface forces: Changing concepts and complexity with dissolved gas, bubbles, salt and heat, Curr. Opin. Colloid Interface Sci., 2017, 27, 25-32.

36. F. Cugia, M. Monduzzi, B.W. Ninham, A. Salis, Interplay of ion specificity, $\mathrm{pH}$ and buffers: insights from electrophoretic mobility and $\mathrm{pH}$ measurements of lysozyme solutions, RSC Advances, 2013, 3(17), 58825888.

37. A. Salis, L. Cappai, C. Carucci, D.F. Parsons, M. Monduzzi, Specific Buffer Effects on the Intermolecular Interactions among Protein Molecules at Physiological pH, J. Phy. Chem. Lett., 2020, 11(16), 68056811. 